Kragujevac Journal of Mathematics

Volume 44(1) (2020), Pages 101-111.

\title{
ON THE LOCAL VERSION OF THE CHERN CONJECTURE: CMC HYPERSURFACES WITH CONSTANT SCALAR CURVATURE IN $\mathbb{S}^{n+1}$
}

\begin{abstract}
S. C. DE ALMEIDA ${ }^{1}$, F. G. B. BRITO ${ }^{2}$, M. SCHERFNER ${ }^{3}$, AND S. WEISS ${ }^{4}$
ABSTRACT. After nearly 50 years of research the Chern conjecture for isoparametric hypersurfaces in spheres is still an unsolved and important problem and in particular its local version is of great interest, since here one loses the power of Stokes' Theorem as a method for proving it. Here we present a related result for CMC hypersurfaces in $\mathbb{S}^{n+1}$ with constant scalar curvature and three distinct principal curvatures.
\end{abstract}

\section{INTRODUCTION}

The Chern conjecture for isoparametric hypersurfaces in spheres can be stated as follows. Let $M$ be a closed, minimally immersed hypersurface of the $(n+1)$-dimensional sphere $\mathbb{S}^{n+1}$ with constant scalar curvature. Then $M$ is isoparametric.

One obvious generalization is that on non-closed manifolds, i.e., a local version of the conjecture. This has in particular been proposed by Bryant for the case $n=3$.

Let $M \subset \mathbb{S}^{4}$ be a minimal hypersurface with constant scalar curvature. Then $M$ is isoparametric.

For more details, a short history and an overview of results we would like to refer to the review article [3] by Scherfner, Weiss and Yau.

Here we will give a result related to the local version.

Let $n>3$ and $M \subset \mathbb{S}^{n+1}$ be a hypersurface with constant mean and scalar curvatures which has three pairwise distinct principal curvatures everywhere, then $M$ is isoparametric.

Key words and phrases. Constant mean and scalar curvature, isoparametric hypersurfaces, Chern conjecture.

2010 Mathematics Subject Classification. Primary: 53C42. Secondary: 53C21.

DOI 10.46793/KgJMat2001.101A

Received: December 25, 2017.

Accepted: February 24, 2018. 


\section{Preliminaries}

Let $M$ be an $n$-dimensional hypersurface in a unit sphere $\mathbb{S}^{n+1}(1)$. We choose a local orthonormal frame field $\left\{e_{1}, \ldots, e_{n+1}\right\}$ in $\mathbb{S}^{n+1}(1)$, so that restricted to $M, e_{1}, \ldots, e_{n}$ are tangent to $M$. Let $\omega_{1}, \ldots, \omega_{n+1}$ denote the dual co-frame field in $\mathbb{S}^{n+1}(1)$. We use the following convention for the indices: $A, B, C, D$ range from 1 to $n+1$ and $i, j, k$ from 1 to $n$. The structure equations of $\mathbb{S}^{n+1}(1)$ as a hypersurface of the Euclidean space $\mathbb{R}^{n+2}$ are given by

$$
\begin{aligned}
d \omega_{A} & =-\sum_{B} \omega_{A B} \wedge \omega_{B}, \quad \omega_{A B}+\omega_{B A}=0 \\
d \omega_{A B} & =-\sum_{C} \omega_{A C} \wedge \omega_{C B}+\frac{1}{2} \sum_{C, D} \bar{R}_{A B C D} \omega_{C} \wedge \omega_{D},
\end{aligned}
$$

where $\bar{R}$ is the Riemannian curvature tensor

$$
\bar{R}_{A B C D}=\delta_{A C} \delta_{B D}-\delta_{A D} \delta_{B C} .
$$

The contractions $\bar{R}_{A C}=\sum_{B} \bar{R}_{A B C B}$ and $\bar{R}=\sum_{A, B} \bar{R}_{A B A B}$ are the Ricci curvature tensor and the scalar curvature of $\mathbb{S}^{n+1}(1)$, respectively. Next, we restrict all the tensors to $M$. First of all, since $\omega_{n+1}=0$ on $M, \sum_{i} \omega_{n+1, i} \wedge \omega_{i}=d \omega_{n+1}=0$. By Cartan's lemma we can write

$$
\omega_{n+1, i}=\sum_{j} h_{i j} \omega_{i}, \quad h_{i j}=h_{j i}
$$

Here $h=\sum_{i, j} h_{i j} \omega_{i} \omega_{j}$ denotes the second fundamental form of $M$ and the principal curvatures $\lambda_{i}$ are the eigenvalues of the matrix $\left(h_{i j}\right)$. Furthermore the mean curvature is given by $H=\frac{1}{n} \sum_{i} h_{i i}=\frac{1}{n} \sum_{i} \lambda_{i}$ and $K=\operatorname{det}\left(h_{i j}\right)=\prod_{i} \lambda_{i}$ is the Gauss-Kronecker curvature. We also define

$$
S:=|h|^{2}=\sum_{i, j} h_{i j}^{2}=\sum_{i} \lambda_{i}^{2}
$$

and for $r \geq 3$

$$
f_{r}:=\operatorname{tr}\left(\left(h_{i j}\right)^{r}\right) .
$$

Independently of the choice of the $e_{i}$ we have

$$
f_{3}=\sum_{i, j, k} h_{i j} h_{j k} h_{k i}=\sum_{i} \lambda_{i}^{3}, \quad f_{4}=\sum_{i, j, k, l} h_{i j} h_{j k} h_{k l} h_{l i}=\sum_{i} \lambda_{i}^{4},
$$

and so on.

On $M$ we have

$$
\begin{aligned}
d \omega_{i} & =-\sum_{j} \omega_{i j} \wedge \omega_{j}, \quad \omega_{i j}+\omega_{j i}=0 \\
d \omega_{i j} & =-\sum_{k} \omega_{i k} \wedge \omega_{k j}+\frac{1}{2} \sum_{k, l} R_{i j k l} \omega_{k} \wedge \omega_{l},
\end{aligned}
$$


where $R$ is the Riemannian curvature tensor on $M$ with components satisfying

$$
0=R_{i j k l}+R_{i j l k}
$$

These structure equations imply the following integrability condition (Gauss equation):

$$
R_{i j k l}=\left(\delta_{i k} \delta_{j l}-\delta_{i l} \delta_{j k}\right)+\left(h_{i k} h_{j l}-h_{i l} h_{j k}\right) .
$$

For the scalar curvature we have

$$
\kappa=n(n-1)+n^{2} H^{2}-S .
$$

If we consider minimal hypersurfaces, the Ricci curvature and scalar curvature are given by, respectively,

$$
\begin{aligned}
R_{i j} & =(n-1) \delta_{i j}-\sum_{k} h_{i k} h_{j k}, \\
\kappa & =n(n-1)-S .
\end{aligned}
$$

It follows from (2.9) that $\kappa$ is constant if and only if $S$ is constant. The covariant derivative $\nabla h$ with components $h_{i j k}$ is given by

$$
\sum_{k} h_{i j k} \omega_{k}=d h_{i j}+\sum_{k} h_{j k} \omega_{i k}+\sum_{k} h_{i k} \omega_{j k}
$$

Then the exterior derivative of (2.8) together with the structure equations yields the following Codazzi equation

$$
h_{i j k}=h_{i k j}=h_{j i k} .
$$

In addition we have

$$
\begin{aligned}
h_{i j k} & =\left(h_{i j}\right)_{k}+\sum_{l} h_{j l} \omega_{i l}\left(e_{k}\right)+\sum_{l} h_{i l} \omega_{j l}\left(e_{k}\right), \\
h_{i j k l} & =\left(h_{i j k}\right)_{l}+\sum_{m} h_{m j k} \omega_{i m}\left(e_{l}\right)+\sum_{m} h_{i m k} \omega_{j m}\left(e_{l}\right)+\sum_{m} h_{i j m} \omega_{k m}\left(e_{l}\right), \\
h_{i j k l} & =h_{i j l k}+\sum_{m} h_{m j} R_{m i k l}+\sum_{m} h_{m i} R_{m j k l}, \\
\sum_{i j k} h_{i j k}^{2} & =(S-n) S-n H f_{3}+n^{2} H^{2} .
\end{aligned}
$$

We will use the following result by Otsuki given in [2].

Lemma 2.1. Let $M$ be a hypersurface in a $(n+1)$-dimensional Riemannian manifold of constant curvature such that the multiplicities of the principal curvatures are all constant. Then the distribution of the space of principal vectors corresponding to each principal curvature is completely integrable. If the multiplicity of a principal curvature is greater than 1, then this principal curvature is constant on each integral submanifold of the corresponding distribution of the space of principal vectors. 


\section{Proof of the Theorem}

Theorem 3.1. Let $n>3$ and $M \subset \mathbb{S}^{n+1}$ be a hypersurface with constant mean and scalar curvatures which has three pairwise distinct principal curvatures everywhere, then $M$ is isoparametric.

Proof. Let $\lambda, \mu$ und $\nu$ be the distinct principal curvatures with corresponding multiplicities $r_{1}, r_{2}$ and $r_{3}$. From $r_{1}+r_{2}+r_{3}=n$ and the definitions of $H$ and $S$ one has a system of equations with continuous coefficients which the $r_{i}$ solve uniquely. Thus the $r_{i}$ are continuous functions and therefore constant.

Locally we choose the $e_{i}$ such that $h$ is diagonal in every point. For the directional derivatives of the principal curvatures one has

$$
r_{1} \lambda_{k}+r_{2} \mu_{k}+r_{3} \nu_{k}=r_{1} \lambda \lambda_{k}+r_{2} \mu \mu_{k}+r_{3} \nu \nu_{k}=0
$$

Let the principal curvature directions corresponding to the three principal curvatures be called $e_{A}, e_{a}$ and $e_{\alpha}$. Then (2.12) implies

$$
h_{i j k}=\delta_{i j}\left(\lambda_{i}\right)_{k} \text {, }
$$

for $\lambda_{i}=\lambda_{j}$ and

$$
\omega_{i j}\left(e_{k}\right)=\frac{1}{\lambda_{j}-\lambda_{i}} h_{i j k}
$$

for $\lambda_{i} \neq \lambda_{j}$.

We consider different cases for the multiplicities of the principal curvatures. Without loss of generality, let $r_{1} \geq r_{2} \geq r_{3}$.

Case 1: $r_{1}, r_{2}, r_{3}>1$. Then Lemma 2.1 implies $\lambda_{A}=\mu_{a}=\nu_{\alpha}=0$, and with (3.1) it follows that all derivatives of the principal curvatures vanish.

Case 2: $r_{1}, r_{2}>1, r_{3}=1$. Without loss of generality let $\alpha=n$. Then Lemma 2.1 and (3.1) imply that the derivatives of the principal curvatures in directions $e_{A}$ and $e_{a}$ vanish. From (3.2), (3.3) and (2.13) one has

$$
\begin{aligned}
h_{A a B a} & =\left(h_{A a B}\right)_{a}+\sum_{m} h_{m a B} \omega_{A m}\left(e_{a}\right)+\sum_{m} h_{A m B} \omega_{a m}\left(e_{a}\right)+\sum_{m} h_{A a m} \omega_{B m}\left(e_{a}\right) \\
& =\frac{2}{\nu-\lambda} h_{a A n} h_{a B n}+\delta_{A B} \frac{\lambda_{n} \mu_{n}}{\nu-\mu}, \\
h_{A a a B} & =\frac{2}{\nu-\mu} h_{a A n} h_{a B n}+\delta_{A B} \frac{\lambda_{n} \mu_{n}}{\nu-\lambda} .
\end{aligned}
$$

From (2.14) one has

$$
h_{A a B a}-h_{A a a B}=(\lambda-\mu) R_{A a B a}=\delta_{A B}(\lambda-\mu)(1+\mu \lambda)
$$

and thus

$$
h_{a A n} h_{a B n}=\frac{z_{1}}{2} \delta_{A B},
$$

where

$$
z_{1}:=(\nu-\lambda)(\nu-\mu)(1+\lambda \mu)+\lambda_{n} \mu_{n} .
$$


Let $v_{a}$ be the column vector of the $h_{a A n}$ for a given $a$, then this can be expressed in the matrix equation

$$
v_{a} v_{a}^{t}=\frac{z_{1}}{2} i d
$$

Since the left hand side can only have rank 0 or 1 , it follows that $z_{1}=0$ and therefore $h_{a A n}=0$ for all $a$ und $A$. From (3.1) it follows that

$$
\lambda_{n}=\frac{1}{r_{1}} \frac{\nu-\mu}{\mu-\lambda} \nu_{n}, \quad \mu_{n}=\frac{1}{r_{2}} \frac{\nu-\lambda}{\lambda-\mu} \nu_{n}
$$

and thus

$$
\begin{aligned}
\sum_{i j k} h_{i j k}^{2} & =3 \sum_{A} h_{A A n}^{2}+3 \sum_{a} h_{a a n}^{2}+h_{n n n}^{2}=3 r_{1} \lambda_{n}^{2}+3 r_{2} \mu_{n}^{2}+\nu_{n}^{2} \\
& =\left(\frac{3}{r_{1}} \frac{(\nu-\mu)^{2}}{(\mu-\lambda)^{2}}+\frac{3}{r_{2}} \frac{(\nu-\lambda)^{2}}{(\lambda-\mu)^{2}}+1\right) \nu_{n}^{2} \\
& =\left(3 r_{2}(\nu-\mu)^{2}+3 r_{1}(\nu-\lambda)^{2}+r_{1} r_{2}(\lambda-\mu)^{2}\right) \frac{1}{r_{1} r_{2}} \frac{1}{(\lambda-\mu)^{2}} \nu_{n}^{2} .
\end{aligned}
$$

On the other hand, $z_{1}=0$ implies

$$
\frac{1}{r_{1} r_{2}} \frac{1}{(\lambda-\mu)^{2}} \nu_{n}^{2}=-\frac{\lambda_{n} \mu_{n}}{(\nu-\mu)(\nu-\lambda)}=1+\lambda \mu
$$

and one has

$$
r_{2}(\nu-\mu)^{2}+r_{1}(\nu-\lambda)^{2}+r_{1} r_{2}(\lambda-\mu)^{2}=\frac{1}{2} \sum_{i j}\left(\lambda_{i}-\lambda_{j}\right)^{2}=n S-n^{2} H^{2} .
$$

Then (2.15) is of the form

$$
(S-n) S+n^{2} H^{2}=(1+\lambda \mu)\left(3 n S-3 n^{2} H^{2}-2 r_{1} r_{2}(\lambda-\mu)^{2}\right)+n H f_{3}
$$

From

$$
r_{1} \lambda+r_{2} \mu+\nu=n H, \quad r_{1} \lambda^{2}+r_{2} \mu^{2}+\nu^{2}=S
$$

one has

$$
r_{1}\left(1+r_{1}\right) \lambda^{2}+r_{2}\left(1+r_{2}\right) \mu^{2}+n^{2} H^{2}-2 n H r_{1} \lambda-2 n H r_{2} \mu+2 r_{1} r_{2} \lambda \mu-S=0 .
$$

Solving for $\lambda$ yields

where

$$
\lambda=\frac{n H-r_{2} \mu}{1+r_{1}}+w
$$

$$
w:= \pm \sqrt{\frac{-n r_{2} \mu^{2}+2 n r_{2} H \mu+\left(1+r_{1}\right) S-n^{2} H^{2}}{r_{1}\left(1+r_{1}\right)^{2}}} .
$$

If $w=0$ on an open set, then $\mu$ and consequently $\lambda$ and $\nu$ are constant there. Therefore it is sufficient to show the proposition under the assumption that the sign of $w$ remains the same. One calculates

$$
1+\lambda \mu=\frac{n H \mu-r_{2} \mu^{2}}{1+r_{1}}+1+w \mu
$$




$$
\begin{aligned}
(\lambda-\mu)^{2}= & \left(n \frac{H-\mu}{1+r_{1}}+w\right)^{2} \\
= & n^{2} \frac{\mu^{2}-2 H \mu+H^{2}}{\left(1+r_{1}\right)^{2}}+2 n \frac{H-\mu}{1+r_{1}} w+w^{2} \\
= & \frac{n^{2} r_{1}-n r_{2}}{r_{1}\left(1+r_{1}\right)^{2}} \mu^{2}+\frac{2 n H\left(r_{2}-n r_{1}\right)}{r_{1}\left(1+r_{1}\right)^{2}} \mu+\frac{S}{r_{1}\left(1+r_{1}\right)} \\
& +\frac{n^{2} H^{2}\left(r_{1}-1\right)}{r_{1}\left(1+r_{1}\right)^{2}}+2 n \frac{H-\mu}{1+r_{1}} w \\
f_{3}= & r_{1} \lambda^{3}+r_{2} \mu^{3}+\nu^{3} \\
= & r_{1}\left(1-r_{1}^{2}\right) \lambda^{3}+r_{2}\left(1-r_{2}^{2}\right) \mu^{3}+n^{3} H^{3}-3 n^{2} H^{2} r_{1} \lambda-3 n^{2} H^{2} r_{2} \mu \\
& +3 n H r_{1}^{2} \lambda^{2}+3 n H r_{2}^{2} \mu^{2}-3 r_{1}^{2} r_{2} \lambda^{2} \mu-3 r_{1} r_{2}^{2} \lambda \mu^{2}+6 n H r_{1} r_{2} \lambda \mu \\
= & \ldots \mu^{3}+\ldots \mu^{2}+\ldots \mu+\ldots+w \mu(\ldots \mu+\ldots),
\end{aligned}
$$

that is

$$
(1+\lambda \mu)\left(3 n S-3 H^{2}-2 r_{1} r_{2}(\lambda-\mu)^{2}\right)+n H f_{3}=P_{1}(\mu)+P_{2}(\mu) w,
$$

where $P_{1}$ and $P_{2}$ are polynomials of constant coefficients. For $P_{2}$ one has

$$
P_{2}(t)=\ldots t^{3}+\ldots t^{2}+\ldots t-\frac{4 n r_{1} r_{2} H}{1+r_{1}},
$$

therefore, it is not identically zero if $H \neq 0$. For the case $H=0$ the same follows from

$$
P_{2}(t)=\ldots t^{3}+\frac{\left(3 n+3 n r_{1}-2 r_{2}\right) S+4 n r_{1} r_{2}}{1+r_{1}} t
$$

with

$$
3 n+3 n r_{1}-2 r_{2} \geq 3 n-2 r_{2} \geq n>0 .
$$

It follows that $w-R(\mu)=0$ for a rational function $R$. The function

$$
F(t):= \pm \sqrt{\frac{-n r_{2} t^{2}+2 n r_{2} H t+\left(1+r_{1}\right) S-n^{2} H^{2}}{r_{1}\left(1+r_{1}\right)^{2}}}-R(t)
$$

is analytical and not constant. $F(\mu)=0$ then implies that $\mu$ is constant. Consequently $\lambda$ and $\nu$ are also constant and the proposition follows.

Case 3: $r_{1}=: r=n-2>1, r_{2}=r_{3}=1$.

Without loss of generality let $a=1$ and $\alpha=n$. Then the derivatives of the principal curvatures in $e_{A}$ direction vanish, and analogously to case 2 one has

$$
\begin{aligned}
& h_{A n B n}=\delta_{A B}\left(\lambda_{n n}+\frac{\lambda_{1} \nu_{1}}{\mu-\nu}\right)+\frac{2}{\mu-\lambda} h_{1 A n} h_{1 B n}, \\
& h_{A n n B}=\delta_{A B}\left(\frac{\nu_{1} \lambda_{1}}{\mu-\lambda}+\frac{\nu_{n} \lambda_{n}}{\nu-\lambda}+\frac{2 \lambda_{n}^{2}}{\lambda-\nu}\right)+\frac{2}{\mu-\nu} h_{1 A n} h_{1 B n}
\end{aligned}
$$


and thus

$$
h_{1 A n} h_{1 B n}=\frac{z_{2}}{2} \delta_{A B},
$$

where

$$
z_{2}:=\lambda_{1} \nu_{1}+\frac{(\mu-\lambda)(\mu-\nu)}{\lambda-\nu}\left((\lambda-\nu)(1+\lambda \nu)-\lambda_{n n}+\frac{\lambda_{n} \nu_{n}}{\nu-\lambda}+\frac{2 \lambda_{n}^{2}}{\lambda-\nu}\right) .
$$

As in case 2 it follows that $h_{1 A n}=0$ for all $A$. From $z_{2}=0$ one has

$$
\begin{aligned}
\lambda_{n n} & =(\lambda-\nu)(1+\lambda \nu)+\frac{\lambda_{n} \nu_{n}}{\nu-\lambda}+\frac{2 \lambda_{n}^{2}}{\lambda-\nu}+\frac{\lambda-\nu}{(\mu-\lambda)(\mu-\nu)} \lambda_{1} \nu_{1} \\
& =(\lambda-\nu)(1+\lambda \nu)-(n-2) \frac{\lambda-\nu}{(\mu-\nu)^{2}} \lambda_{1}^{2}+\frac{(n+1) \mu-\nu-n H}{(\lambda-\nu)(\mu-\nu)} \lambda_{n}^{2}
\end{aligned}
$$

and in the same way it follows that

$$
\lambda_{11}=(\lambda-\mu)(1+\lambda \mu)+\frac{(n+1) \nu-\mu-n H}{(\lambda-\mu)(\nu-\mu)} \lambda_{1}^{2}-(n-2) \frac{\lambda-\mu}{(\nu-\mu)^{2}} \lambda_{n}^{2} .
$$

From $h_{a 1 a n}-h_{a 1 n a}=0$ one has

$$
\lambda_{1 n}=\frac{(n-2)(\lambda-\mu)^{2}(\lambda-\nu)+n(n-1)(\mu-\nu)^{2}(\lambda-H)}{(\mu-\nu)^{2}(\lambda-\mu)(\lambda-\nu)} \lambda_{1} \lambda_{n}
$$

and again the same holds true for reversed indices:

$$
\lambda_{n 1}=\frac{(n-2)(\lambda-\nu)^{2}(\lambda-\mu)+n(n-1)(\mu-\nu)^{2}(\lambda-H)}{(\mu-\nu)^{2}(\lambda-\mu)(\lambda-\nu)} \lambda_{1} \lambda_{n} .
$$

(2.15) is of the form

$$
\begin{aligned}
|\nabla h|^{2}= & 3(n-2) \lambda_{1}^{2}+\mu_{1}^{2}+3 \nu_{1}^{2}+3(n-2) \lambda_{n}^{2}+3 \mu_{n}^{2}+\nu_{n}^{2} \\
= & \left(3(n-2)+(n-2)^{2} \frac{(\lambda-\nu)^{2}}{(\nu-\mu)^{2}}+3(n-2)^{2} \frac{(\lambda-\mu)^{2}}{(\mu-\nu)^{2}}\right) \lambda_{1}^{2} \\
& +\left(3(n-2)+3(n-2)^{2} \frac{(\lambda-\nu)^{2}}{(\nu-\mu)^{2}}+(n-2)^{2} \frac{(\lambda-\mu)^{2}}{(\mu-\nu)^{2}}\right) \lambda_{n}^{2},
\end{aligned}
$$

that is

$$
\begin{aligned}
(\nu-\mu)^{2}|\nabla h|^{2}= & \left(3(n-2)\left(n S-H^{2}\right)-2(n-2)^{2}(\lambda-\nu)^{2}\right) \lambda_{1}^{2} \\
& +\left(3(n-2)\left(n S-H^{2}\right)-2(n-2)^{2}(\lambda-\mu)^{2}\right) \lambda_{n}^{2} .
\end{aligned}
$$

If $\lambda_{1}=0$ on an open set, (3.8) and (3.11) imply

$$
|\nabla h|^{2}=\left(3 n S-3 H^{2}-2(n-2)(\lambda-\mu)^{2}\right)(1+\lambda \mu)
$$

and as in case 2 it follows that the principal curvatures are constant. The same holds true for $\lambda_{n}=0$, therefore we can presume in the following that $\lambda_{1} \neq 0$ and $\lambda_{n} \neq 0$. Deriving (3.11) in direction $e_{1}$ yields

$$
2(\nu-\mu)\left(\nu_{1}-\mu_{1}\right)|\nabla h|^{2}+(\nu-\mu)^{2}\left(|\nabla h|^{2}\right)_{1}
$$




$$
\begin{aligned}
= & -4(n-2)^{2}(\lambda-\nu)\left(\lambda_{1}-\nu_{1}\right) \lambda_{1}^{2} \\
& +2\left(3(n-2)\left(n S-H^{2}\right)-2(n-2)^{2}(\lambda-\nu)^{2}\right) \lambda_{1} \lambda_{11} \\
& -4(n-2)^{2}(\lambda-\mu)\left(\lambda_{1}-\mu_{1}\right) \lambda_{n}^{2} \\
& +2\left(3(n-2)\left(n S-H^{2}\right)-2(n-2)^{2}(\lambda-\mu)^{2}\right) \lambda_{n} \lambda_{n 1}
\end{aligned}
$$

and with

$$
\begin{aligned}
\nu_{1}-\mu_{1} & =n(n-2) \frac{\lambda-H}{\mu-\nu} \lambda_{1} \\
\left(|\nabla h|^{2}\right)_{1} & =-n H\left(f_{3}\right)_{1}=-3 n(n-2) H(\lambda-\mu)(\lambda-\nu) \lambda_{1}
\end{aligned}
$$

one has

$$
\begin{aligned}
& n|\nabla h|^{2}(H-\lambda)-\frac{3 n}{2} H(\mu-\nu)^{2}(\lambda-\mu)(\lambda-\nu) \\
= & -2 n(n-2)(\mu-H) \frac{\lambda-\nu}{\mu-\nu} \lambda_{1}^{2}-2 n(n-2)(\nu-H) \frac{\lambda-\mu}{\nu-\mu} \lambda_{n}^{2} \\
& +\left(3 n S-3 H^{2}-2(n-2)(\lambda-\nu)^{2}\right) \lambda_{11} \\
& +\left(3 n S-3 H^{2}-2(n-2)(\lambda-\mu)^{2}\right) \frac{\lambda_{n}}{\lambda_{1}} \lambda_{n 1} .
\end{aligned}
$$

To simplify notation, we set

$$
A_{1}(x, y)=3\left(n S-H^{2}\right)-2(n-2)(x-y)^{2} .
$$

Putting (3.8) and (3.10) into (3.12) we have

$$
\begin{aligned}
& n|\nabla h|^{2}(H-\lambda)-\frac{3 n}{2} H(\mu-\nu)^{2}(\lambda-\mu)(\lambda-\nu)-A_{1}(\lambda, \nu)(\lambda-\mu)(1+\lambda \mu) \\
= & \left(-2 n(n-2)(\mu-H) \frac{\lambda-\nu}{\mu-\nu}+A_{1}(\lambda, \nu) \frac{(n+1) \nu-\mu-n H}{(\lambda-\mu)(\nu-\mu)}\right) \lambda_{1}^{2} \\
& -\left(2 n(\nu-H) \frac{\lambda-\mu}{\nu-\mu}+\frac{3 n S-3 H^{2}+2(n-2)(\lambda-\mu)(\lambda-\nu)}{\mu-\nu}\right. \\
& \left.-\frac{n(n-1)(\lambda-H)}{(n-2) \lambda-\mu)(\lambda-\nu)} A_{1}(\lambda, \mu)\right)(n-2) \lambda_{n}^{2} .
\end{aligned}
$$

Analogously we have

$$
\begin{aligned}
& n|\nabla h|^{2}(H-\lambda)-\frac{3 n}{2} H(\mu-\nu)^{2}(\lambda-\mu)(\lambda-\nu)-A_{1}(\lambda, \mu)(\lambda-\nu)(1+\lambda \nu) \\
= & \left(-2 n(n-2)(\nu-H) \frac{\lambda-\mu}{\nu-\mu}+A_{1}(\lambda, \mu) \frac{(n+1) \mu-\nu-n H}{(\lambda-\nu)(\mu-\nu)}\right) \lambda_{n}^{2} \\
& -\left(2 n(\mu-H) \frac{\lambda-\nu}{\mu-\nu}+\frac{3 n S-3 H^{2}+2(n-2)(\lambda-\mu)(\lambda-\nu)}{\nu-\mu}\right. \\
& \left.-\frac{n(n-1)(\lambda-H)}{(n-2)(\lambda-\mu)(\lambda-\nu)} A_{1}(\lambda, \nu)\right)(n-2) \lambda_{1}^{2} .
\end{aligned}
$$


From (3.11) one has

$$
(n-2) \lambda_{n}^{2}=\frac{(\nu-\mu)^{2}|\nabla h|^{2}}{A_{1}(\lambda, \mu)}-\frac{(n-2) \varepsilon(\nu)}{A_{1}(\lambda, \mu)} \lambda_{1}^{2} .
$$

As in case 2

$$
(n-2) \lambda+\mu+\nu=n H, \quad(n-2) \lambda^{2}+\mu^{2}+\nu^{2}=S,
$$

yield

$$
\lambda=\frac{1}{n-1}(n H-\nu)-\frac{1}{n-2} w, \quad \mu=\frac{1}{n-1}(n H-\nu)+w
$$

with

$$
w:= \pm \frac{\sqrt{n-2}}{n-1} \sqrt{-n \nu^{2}+2 n H \nu+(n-1) S-n^{2} H^{2}},
$$

where again the sign of $w$ can be assumed to remain the same. We set

$$
\varepsilon(\lambda, \mu, \nu)=A_{1}(\lambda, \mu) A_{2}(\lambda, \mu, \nu) A_{3}(\lambda, \mu, \nu)+A_{1}(\lambda, \nu) A_{2}(\lambda, \nu, \mu) A_{3}(\lambda, \nu, \mu),
$$

where $A_{1}$ was already defined by

$$
A_{1}(x, y):=3 n S-3 H^{2}-2(n-2)(x-y)^{2},
$$

and $A_{2}$ and $A_{3}$ given by

$$
\begin{aligned}
A_{2}(x, y, z):= & (y-z)(x-z)\left(n(n-2)|\nabla h|^{2}(x-H)(x-y) A_{1}(x, z)\right. \\
& +\frac{3}{2} n(n-2) H(y-z)^{2}(x-y)^{2}(x-z) A_{1}(x, z) \\
& +(n-2)(1+x y)(x-y)^{2} A_{1}(x, z)^{2} \\
& +2 n(n-2)|\nabla h|^{2}(y-H)(x-y)(x-z)(z-y) \\
& \left.+|\nabla h|^{2}((n+1) z-y-n H)(z-y) A_{1}(x, z)\right), \\
A_{3}(x, y, z):= & -2(n-2)^{2} n(y-H)(x-y)(x-z)^{2} A_{1}(x, y) \\
& +(n-2)^{2}(x-y)(x-z)\left(3 n S-3 H^{2}+2(n-2)(x-y)(x-z)\right) A_{1}(x, y) \\
& +n(n-1)(n-2)(x-H)(y-z) A_{1}(x, y) A_{1}(x, z) \\
& -2 n(n-2)^{2}(z-H)(x-y)^{2}(x-z) A_{1}(x, z) \\
& -(n-2)(x-y)((n+1) y-z-n H) A_{1}(x, y) A_{1}(x, z) .
\end{aligned}
$$

From (3.13), (3.14) and (3.15) one has the following condition for $\lambda, \mu$ and $\nu$ :

$$
\varepsilon(\lambda, \mu, \nu)=|\nabla h|^{2}(\mu-\nu)^{2} A_{3}(\lambda, \mu, \nu) A_{3}(\lambda, \nu, \mu) .
$$

Using (3.16) and (3.17) the terms in (3.18) can be written as polynomials in $\nu$ and $w$ whose leading coefficients are given by

$$
\begin{aligned}
& A_{1}(\lambda, \mu)=2 n \nu^{2}-4 n H \nu+(n+2) S+\left(2 n^{2}-3\right) H^{2} \\
& A_{1}(\lambda, \nu)=-2 \frac{(n-2) n^{2}-n}{(n-1)^{2}} \nu^{2}+\cdots-\left(\frac{4 n}{n-1} \nu+\cdots\right) w
\end{aligned}
$$




$$
\begin{aligned}
A_{2}(\lambda, \mu, \nu)= & -\frac{8 n^{4}\left(7 n^{6}-42 n^{5}+57 n^{4}+44 n^{3}-79 n^{2}-18 n-1\right)}{(n-2)(n-1)^{8}} \nu^{10}+\cdots \\
& +\left(\frac{8 n^{4}\left(n^{6}-6 n^{5}-9 n^{4}+68 n^{3}-41 n^{2}-46 n+1\right)}{(n-2)(n-1)^{7}} \nu^{9}+\cdots\right) w, \\
A_{2}(\lambda, \nu, \mu)= & \frac{8 n^{4}\left(3 n^{2}-6 n+1\right)}{(n-1)^{4}} \nu^{10}+\cdots+\left(\frac{8 n^{4}\left(n^{2}-2 n+3\right)}{(n-1)^{3}} \nu^{9}+\cdots\right) w, \\
A_{3}(\lambda, \mu, \nu)= & -\frac{2(n-2) n^{3}\left(7 n^{3}-17 n^{2}-19 n+1\right)}{(n-1)^{3}} \nu^{6}+\cdots \\
& +\left(-\frac{2 n^{2}\left(12 n^{3}-20 n^{2}+3 n+1\right)}{(n-1)^{2}} \nu^{5}+\cdots\right) w, \\
A_{3}(\lambda, \nu, \mu)= & \frac{8(n-2) n^{3}\left(n^{4}-4 n^{3}-2 n^{2}+12 n+1\right)}{(n-1)^{4}} \nu^{6}+\cdots \\
& +\left(\frac{4 n^{3}\left(6 n^{4}-9 n^{3}-25 n^{2}+29 n+15\right)}{(n-1)^{3}} \nu^{5}+\cdots\right) w .
\end{aligned}
$$

(3.18) is then of the form

$$
Q_{1}(\nu)+Q_{2}(\nu) w=0,
$$

for polynomials $Q_{1}$ and $Q_{2}$ with constant coefficients. The leading coefficient of $Q_{1}$ is given by

$$
\begin{aligned}
Q_{1}(t)= & \frac{32(n-2) n^{8}}{(n-1)^{11}}\left(73 n^{10}-709 n^{9}+2273 n^{8}-1255 n^{7}-7101 n^{6}+12067 n^{5}\right. \\
& \left.-1089 n^{4}-6461 n^{3}+1048 n^{2}+134 n-4\right) t^{18}+\cdots,
\end{aligned}
$$

therefore, $Q_{1}$ is not identically zero. One then has from (3.19) that $w=R(\nu)$ for a rational function $R$ or that $Q_{1}(\nu)=0$; in both cases the proposition follows.

Acknowledgements. This work was partially supported by UFC and Universidade Federal do ABC.

\section{REFERENCES}

[1] S. Chang, On closed hypersurfaces of constant scalar curvatures and mean curvatures in $S^{n+1}$, Pacific J. Math. 165 (1994), 67-76.

[2] T. Otsuki, Minimal hypersurfaces in a Riemannian manifold of constant curvature, Amer. J. Math. 92 (1970), 145-173.

[3] M. Scherfner, S.-T. Yau and S. Weiss, A review of the Chern conjecture for isoparametric hypersurfaces in Spheres, in: Advances in Geometric Analysis, Advanced Lectures in Mathematics (ALM) 21, International Press, Somerville, 2012, 175-187. 
${ }^{1}$ CAEn, Universidade Federal do Ceará, Av. DA UnIVERSIDAde, 2700 - $2^{o}$ ANDAR - BenfiCA, 60020-181 ForTALEZA-CE,

BRAZIL

Email address: sebastc@caen.ufc.br

${ }^{2}$ Centro de Matemática, Computação e Cognição, UNIVERSIDADE FEDERAL DO ABC, 09.210-170 SANTO ANDRÉ,

BRAZIL

Email address: fabiano.brito@ufabc.edu.br

${ }^{3}$ Anhalt University of Applied Sciences, Computer Science And LANGuAges,

LOHMANNSTR. 23,

06366 KÖthen, Germany

Email address: mike.scherfner@hs-anhalt.de

${ }^{4}$ BERLIN,

GERMANY 